\title{
Mineral dust in PMIP simulations: A short review
}

\author{
Fabrice Lambert ${ }^{1,2,3}$ and Samuel Albani ${ }^{4}$ \\ We review the advances in paleoclimatic dust simulations and describe recent developments and possible future \\ directions for the paleoclimatic dust community within the Paleoclimate Modelling Intercomparison Project.
}

\begin{abstract}
Mineral dust aerosols (hereafter "dust") are an important component of the climate system. Airborne dust particles are usually smaller than $20 \mu \mathrm{m}$ and both scatter and absorb incoming solar radiation as well as outgoing thermal radiation, thus, directly altering Earth's radiative balance. Dust particles can also act as ice and cloud condensation nuclei altering cloud lifetime, or darken snowy surfaces after deposition, thus affecting planetary and surface albedo. Finally, dust particles are composed of various minerals, some of which play an important role in biogeochemical cycles both on land and in the ocean. This mineral makeup also determines the impact of dust on radiation and clouds (Maher et al. 2010).
\end{abstract}

Deserts and semi-arid regions are the main sources of dust to the atmosphere. They are heterogeneously distributed throughout the world, with the largest sources in the subtropics. Dust particles are entrained in the atmosphere by surface winds and reach the higher levels of the troposphere through ascending air currents, and from there they can be transported across the globe. Dust particles are removed from the air by both dry (gravitational settling) and wet (washout through precipitation) deposition processes. Local atmospheric dust concentration and surface deposition therefore depend on the distance to the source, source emission strength, wind speed and direction, and the hydrological cycle. They are also not constant throughout the year, but depend on emission event and washout frequency (Prospero et al. 2002).

Unlike well-mixed greenhouse gases, the climatic effects of dust vary seasonally and regionally and are not well represented by global averages. Close to the source regions, particle concentrations are very high, and can be associated with strong surface direct radiative effects of over $50 \mathrm{~W} / \mathrm{m}^{2}$. The net effect at the top of the atmosphere can be positive or negative, depending on the ratio of small and large particles, the height of the dust layers, particle mineralogy, and the albedo of the underlying surface (Albani and Mahowald 2019; Maher et al. 2010). Over micronutrient-limited regions of the oceans, dust particles are an important source of minerals like iron, and can thus modulate the strength of the biological pump and affect the global carbon cycle (Hain et al. 2014; Lambert et al. 2021).

\section{Dust in PMIP simulations}

Interest in dust as an important aerosol with significant orbital- and millennial-scale variability and potential climate feedbacks emerged in the 1970s and 1980s and soon found its way into the climate modeling community. The first global dust simulations for the Last Glacial Maximum (LGM) were performed in the early 1990s (Joussaume 1993). Over the next three decades, our understanding of the dust cycle improved, thanks to more abundant observations from modern platforms and paleoclimate records (Maher et al. 2010). This allowed for improvements in climate models and their embedded dust schemes, with new observational data, data syntheses, and model development spurring each other on (Albani et al. 2015; Maher et al. 2010; Mahowald et al. 2006).

The paleoclimate dust community has strongly focused on the LGM period, owing to the large dust flux increase marked particularly in mid-and high-latitude paleoarchives. However, only a few modeling groups have tried to simulate the LGM dust cycle, with a varying level of validation against modern and paleodata. Estimates of dust emissions, load, direct radiative effects, and impacts on the carbon cycle through iron fertilization are summarized in Figure 1. The large spread in results can mainly be attributed to differences in the representation of dust emission and deposition mechanisms, differences in boundary conditions (including vegetation), inclusion of glaciogenic (formed by glacier abrasion) dust sources, different aerosol size ranges and optical properties, and assumptions about dustborne iron solubility and bioavailability.

a. Global dust emissions

Overall, the central estimates from these simulations suggest that global LGM dust probably doubled in load compared to the late Holocene. This likely contributed about 25\% (-20 ppmv) to the $\mathrm{CO}_{2}$ drawdown through iron fertilization of the oceans and had a direct radiative forcing of $-0.6 \mathrm{~W} / \mathrm{m}^{2}$, slightly lower than the main other forcing mechanisms (greenhouse gases: $-2.8 \mathrm{~W} / \mathrm{m}^{2}$, ice sheets and sea level changes: $-3.0 \mathrm{~W} / \mathrm{m}^{2}$; Albani et al. 2018). However, direct radiative forcing estimates of global dust average both positive and negative values; locally and regionally, the magnitudes can be much stronger (Albani and Mahowald 2019).

The mid-Holocene (MH) had received far less attention until recently, when it was found that marine sediment records indicated that North African dust emissions were two to five times lower during the "green Sahara" phase than during the late Holocene. These findings motivated the first efforts to simulate and reconstruct the $\mathrm{MH}$ global dust cycle (Albani et al. 2015). New idealized and realistic experiments quickly followed, highlighting the role of dust on the ITCZ and monsoon dynamics (e.g. Albani and Mahowald 2019; Braconnot et al. 2021; Hopcroft et al. 2019).

Although the aforementioned simulations were performed using PMIP climate models (or adaptations thereof), it was only recently that CMIP/PMIP protocols started to include

b. Global dust load

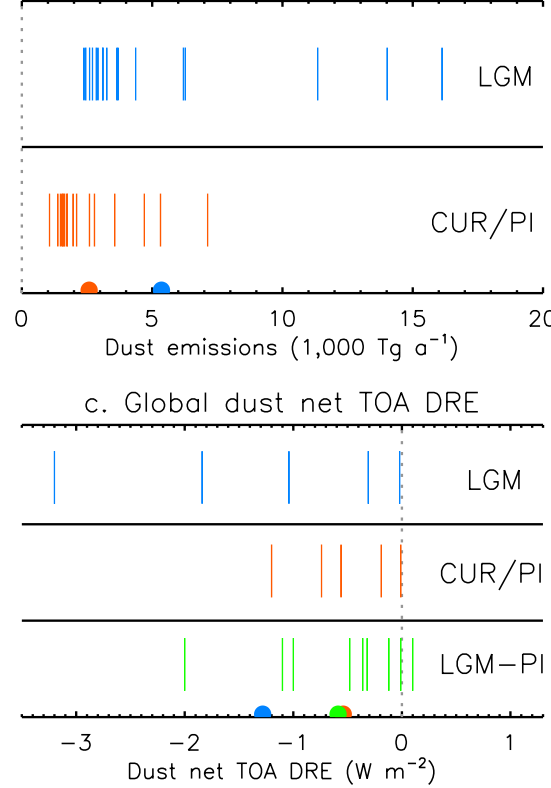

Figure 1: Synthesis of global metrics from LGM dust simulations, adapted from Albani et al. (2018). Vertical bars represent the results of individual experiments. The semi-circles on the $\mathrm{x}$-axes mark the average of the respective model ensembles. The vertical gray dotted lines mark the zero value on the $x$-axis. CUR/PI indicates either current or pre-industrial simulations. TOA DRE stands for Top Of Atmosphere Direct Radiative Effect. 
dust forcings beyond the use of prescribed pre-industrial (PI) or present day (PD) fields. The importance of replacing the PI and PD fields with period-accurate fields (including additional glaciogenic sources in LGM simulations) is evidenced in Figure 2, with LGM surface dust depositions generally several orders of magnitude larger than during the $\mathrm{PI}$ period. Although the inclusion of glaciogenic sources in LGM simulations may not appear crucial for global radiative forcing, they are very important for local and indirect effects (Lambert et al. 2021).

The new CMIP6/PMIP4 protocol allows for dust to vary across climates, either as a prognostically emitted species, or prescribed based on previous paleoclimate simulations or reconstructions (Kageyama et al. 2017; 2018; Otto-Bliesner et al. 2017). As the first PMIP4 papers focused on dust begin to emerge (Braconnot et al. 2021), these efforts are leading to a new exciting phase. We hope that soon many more groups will start to contribute to the effort of understanding the role of dust in the climate system, both as a tracer of past changes of land-surface and atmospheric conditions, as well as an active agent affecting local, regional, and global climate in various ways.

\section{Future Directions}

Recent studies have investigated more complex and detailed dust-climate interactions. These include the dust-vegetation-monsoon nexus (Hopcroft et al. 2019), the effects of dust on snow albedo (Albani and Mahowald 2019; Mahowald et al. 2006; Ohgaito et al. 2018), and the regional features of dust on biogeochemistry, radiative effects and forcing, as well as the dynamical response of the climate system to these forcings (Albani and Mahowald 2019; Braconnot et al. 2021; Lambert et al. 2021).

The newest generation of climate models feature developments of great interest for paleoclimatic dust simulations. These include the incorporation of more realistic particle sizes and optical properties (Albani and Mahowald 2019; Hopcroft et al. 2019) and indirect effects on clouds (Ohgaito et al. 2018), as well as coupling of dust to ocean biogeochemistry, iron processing during atmospheric transport, and explicit representation of particle mineralogy (Hamilton et al. 2019). In its latest iteration, PMIP has been expanding from the mainstay MH and LGM periods to include further equilibrium simulations and transient simulations. Transient simulations are of particular interest to the dust community to investigate the variability and timing of occasional abrupt variations recorded in dust records. These were shown to potentially affect the global carbon cycle on short timescales (Lambert et al. 2021) and additional short-term feedbacks are likely.

To meet future challenges as a community, it is important to highlight the need for interaction and cooperation between the empirical and modeling communities. We stress the need for feedback between the two for project planning. Data syntheses are

a) LGMglacio vs PI

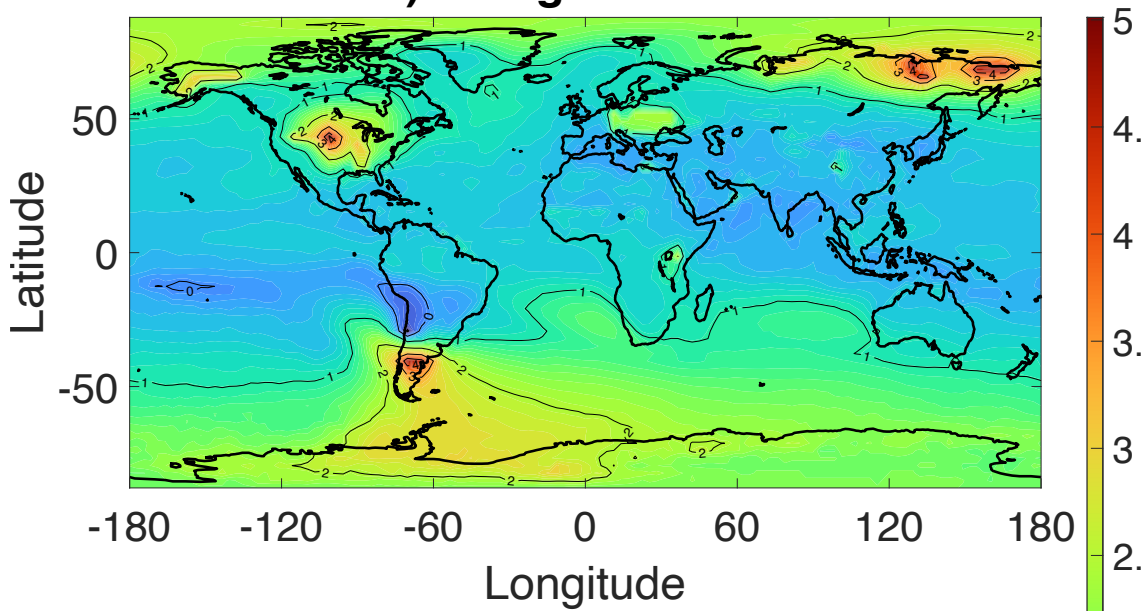

b) LGMglacio vs LGM

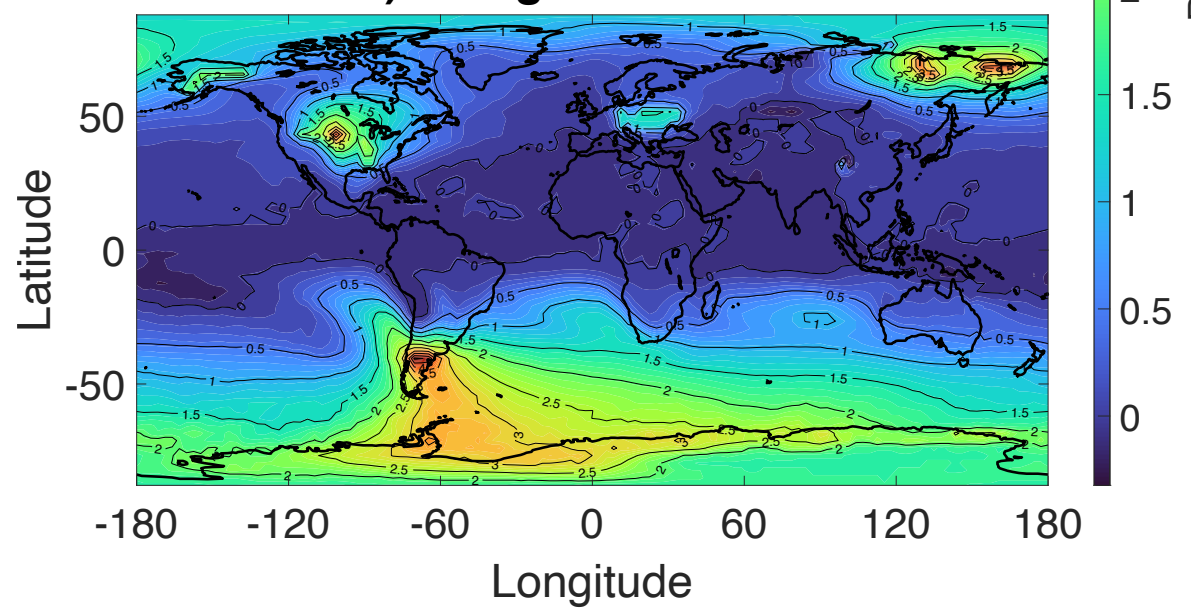

Figure 2: Simulated surface dust deposition ratios (Ohgaito et al. 2018). (A) Ratio of the LGM simulation including glaciogenic sources to the PI simulation; (B) ratio of the LGM simulations with and without glaciogenic sources (note the logarithmic ratio scale).

an important and necessary bridge between empirical measurements and simulations. Ongoing work is expanding the existing Holocene dust synthesis (Albani et al. 2015) to provide a comprehensive dust database of timeseries of dust-mass accumulation rates, with information about the particlesize distribution, over the last glacial-interglacial cycle; it is hoped that this will address some of the needs of the paleoclimate modeling community.

Recently, a new PMIP focus group on dust was launched, with the aims to: (1) coordinate a dust synthesis from PMIP4 experiments and (2) promote the definition of the experimental design for CMIP7/PMIP5 dust experiments. The group may also provide data as a benchmarking tool and boundary conditions for future equilibrium and transient simulations, should this be aligned with the scopes of the next phase. Interested parties are encouraged to contact the authors to participate in this effort.

\section{AFFILIATIONS}

'Department of Physical Geography, Pontificia Universidad Católica de Chile, Santiago, Chile ${ }^{2}$ Millennium Nucleus Paleoclimate, University of Chile, Santiago, Chile

${ }^{3}$ Center for Climate and Resilience Research, University of Chile, Santiago, Chile

${ }^{4}$ Department of Environmental and Earth Sciences, Università degli Studi di Milano-Bicocca, Milan, Italy

\section{CONTACT}

Fabrice Lambert: lambert@uc.cl

\section{REFERENCES}

Albani S, Mahowald NM (2019) J Clim 32: 7897-7913

Albani S et al. (2015) Clim Past 11: 869-903

Albani S et al. (2018) Curr Clim Change Rep 4: 99-114 Braconnot P et al. (2021) Clim Past 17: 1091-1117 Hain MP et al. (2014) In: Holland H, Turekian K (Eds)

Treatise on Geochemistry. Elsevier, 485-517 Hamilton DS et al. (2019) Geosci Model Dev 12: 3835-3862

Hopcroft PO, Valdes PJ (2019) Geophys Res Lett 46: $1612-1621$

Joussaume S (1993) J Geophys Res Atmos 98: 2767-2805

Kageyama M et al. (2017) Geosci Model Dev 10: 4035-4055

Kageyama M et al. (2018) Geosci Model Dev 11: 1033-1057

Lambert F et al. (2021) Earth Planet Sci Lett 554: 116675 Maher BA et al. (2010) Earth Sci Rev 99: 61-97 Mahowald NM et al. (2006) J Geophys Res 111: D10202 Ohgaito Ret al. (2018) Clim Past 14: 1565-1581 Otto-Bliesner BL et al. (2017) Geosci Model Dev 10: 3979-4003

Prospero JM et al. (2002) Rev Geophys 40: 1002 